\title{
Thrombospondin-2 acts as a bridge between tumor extracellular matrix and immune infiltration in pancreatic adenocarcinoma and stomach adenocarcinoma: An integrative pan-cancer analysis
}

Shiyun Tan (D812328105@qq.com )

Renmin Hospital of Wuhan University

Xingchen Liao

Renmin Hospital of Wuhan University

Wei Wang

East Hospital, Renmin Hospital of Wuhan University

Baoping Yu

Renmin Hospital of Wuhan University

\section{Research Article}

Keywords: Thrombospondin-2, pan-cancer, extracellular matrix, immune infiltration, tumor microenvironment, prognosis, biomarker

Posted Date: January 27th, 2022

DOI: https://doi.org/10.21203/rs.3.rs-1288390/v1

License: (c) (1) This work is licensed under a Creative Commons Attribution 4.0 International License. Read Full License 


\section{Abstract}

\section{Background}

Thrombospondin-2 (THBS2) is a versatile glycoprotein that regulates numerous biological functions, including the apoptosis-proliferation balance in endothelial cells, and it has been linked to tumor angiogenesis. However, the role of THBS2 in human cancer remains unknown. This study aimed to determine THBS2 expression in pan-cancer, understand whether THBS2 is associated with its prognosis, and to identify possible roles of THBS2 in tumor immunity and the extracellular matrix (ECM).

\section{Methods}

Data about THBS2 expression in cancers and normal tissues were downloaded from the GenotypeTissue Expression (GTEx) and UCSC Xena and analyzed using the ONCOMINE database, Perl programming language, and the Gene Expression Profiling and Interactive Analyses vision 2 (GEPIA2) web server. Survival prognosis was analyzed using the survival, survminer, and forestplot packages in R $\mathrm{v}$. 4.0.3 and the limma package in R. Immune and matrix components were also analyzed using R v. 4.0.3. Most importantly, we partially validated the role and mechanism of THBS2 in pancreatic and gastric cancers in vitro using PANC1 and BGC-823 cell lines.

\section{Results}

Thrombospondin-2 was significantly overexpressed in 17 of the 33 investigated cancers, and linked to a poor prognosis in pan-cancer survival analysis, especially in adrenocortical (ACC), kidney renal papillary cell (KIRP), and stomach (STAD) carcinomas, kidney chromophobe (KICH), and pancreatic adenocarcinoma (PAAD). Immune infiltration and THBS2 expression were also related. The expression of THBS2 has been linked to immune scores, stromal scores, and immune checkpoint markers in various cancers. The PPI network revealed that THBS2 is associated with multiple extracellular matrix proteins and immune proteins. Knockdown of THBS2 decreased the expression of CD47 and MMP-2 as well as the proliferation, migration, and invasion of PANC1 and BGC-823 cells in vitro.

\section{Conclusions}

Our findings suggested that THBS2 could be a prognostic biomarker that acts as a bridge between the ECM and immune infiltration in various types of cancers, and that it promoted the progression of numerous types of cancers, especially PAAD and STAD.

\section{Background}

Thrombospondin-2 (THBS2) is an extracellular matrix (ECM) protein belonging to the THBS family. Stromal fibroblasts, endothelial cells, and immune cells secrete THBS2, which functions in cell migration, angiogenesis, apoptosis, and cytoskeletal regulation [1]. The significantly elevated expression of THBS2 in various types of cancers, including pancreatic and gastric cancers, is associated with their diagnosis, 
stage, and prognosis. Andreas et al. [2] found that early pancreatic cancer can be screened using serum markers, including THBS2. Li [3] found that combining THBS2 with CA19-9 improved the detection of early gastric cancer. Thrombospondin-2 is also a potential prognostic biomarker in colorectal, pancreatic, and gastric cancers [4].

Interplay between tumors and the immune system is complex. The tumor microenvironment (TME) is crucial for tumorigenesis and progression in human cancers and has recently become a hotspot in tumor research. In addition to cellular components, the TME contains non-cellular components that are dominated by infiltrating immune cells [5] comprising tumor-associated macrophages (TAMs), B cells, CD4+ $T$ and CD8+ $T$ cells, and neutrophils. In addition to mediating immune escape of tumor cells, tumor angiogenesis, and metastasis[6-8], TAMs can affect the prognosis of patients and the effect of immunotherapy $[9,10]$. Compared with the limitations of traditional anticancer therapies, alternative immunotherapy has achieved good results in various cancers [11-13]. However, the relevance of THBS2 in tumor immunity and the underlying mechanisms remain unclear.

We initially acquired information about the differential expression of THBS2 in normal and tumor tissues, then analyzed the relationship between THBS2 expression and patient prognosis in 33 tumors from various aspects. We also explored correlations between THBS2 expression and ECM and tumor immunity. Finally, we knocked down THBS2 expression in PANC1 and BGC-823 cells in vitro to verify our results. Our findings suggested that THBS2 affects the occurrence and development of cancers and the prognosis of patients via interaction with the ECM and immune cells, which might provide suggestions for cancer prevention and treatment targets.

\section{Materials And Methods}

\section{Abbreviations and names of 33 types of cancer.}

The details see Table 1.

Table 1

Abbreviations and names of 33 types of cancer. 
Cancer Abbreviation

ACC Adrenocortical carcinoma LUSC $\begin{aligned} & \text { Lung squamous cell } \\ & \text { carcinoma }\end{aligned}$

\begin{tabular}{|c|c|c|c|}
\hline BLCA & Bladder Urothelial Carcinoma & MESO & Mesothelioma \\
\hline BRCA & Breast invasive carcinoma & OV & $\begin{array}{l}\text { Ovarian serous } \\
\text { cystadenocarcinoma }\end{array}$ \\
\hline CESC & $\begin{array}{l}\text { Cervical squamous cell carcinoma and } \\
\text { endocervical adenocarcinoma }\end{array}$ & PAAD & $\begin{array}{l}\text { Pancreatic } \\
\text { adenocarcinoma }\end{array}$ \\
\hline $\mathrm{CHOL}$ & Cholangio carcinoma & PCPG & $\begin{array}{l}\text { Pheochromocytoma and } \\
\text { Paraganglioma }\end{array}$ \\
\hline COAD & Colon adenocarcinoma & PRAD & Prostate adenocarcinoma \\
\hline DLBC & $\begin{array}{l}\text { Lymphoid Neoplasm Diffuse Large B-cell } \\
\text { Lymphoma }\end{array}$ & READ & Rectum adenocarcinoma \\
\hline ESCA & Esophageal carcinoma & SARC & Sarcoma \\
\hline GBM & Glioblastoma multiforme & SKCM & Skin Cutaneous Melanoma \\
\hline HNSC & Head and Neck squamous cell carcinoma & STAD & Stomach adenocarcinoma \\
\hline $\mathrm{KICH}$ & Kidney Chromophobe & TGCT & $\begin{array}{l}\text { Testicular Germ Cell } \\
\text { Tumors }\end{array}$ \\
\hline KIRC & Kidney renal clear cell carcinoma & THCA & Thyroid carcinoma \\
\hline KIRP & Kidney renal papillary cell carcinoma & THYM & Thymoma \\
\hline LAML & Acute Myeloid Leukemia & UCEC & $\begin{array}{l}\text { Uterine Corpus Endometrial } \\
\text { Carcinoma }\end{array}$ \\
\hline LGG & Brain Lower Grade Glioma & UCS & Uterine Carcinosarcoma \\
\hline LIHC & Liver hepatocellular carcinoma & UVM & Uveal Melanoma \\
\hline LUAD & Lung adenocarcinoma & & \\
\hline
\end{tabular}

\section{Gene expression analysis}

The mRNA expression of THBS2 in different cancers was analyzed using the ONCOMINE database (www.oncomine.org). The expression of THBS2 in different normal tissues and cancers was acquired from GEPIA2 (http://gepia2.cancer-pku.cn/).

\section{Pan-cancer dataset source and processing}


Gene-expression data and full clinical annotation of 33 cancer datasets were obtained from UCSC Xena (https://xenabrowser.net.). We then used Perl software (version 5.34.0) (https://www.perl.org/) to sort out the matrix data of gene expression values.

\section{Survival prognosis}

Survival and prognosis were analyzed using the survival, survminer, and forestplot packages in R v. 4.0.3 and the Bioconductor package limma in R.

\section{Immune infiltration}

We analyzed immune and matrix components in the TME using the estimate package in R (version 4.0.3), and ImmuneScore, StromalScore, and EstimatScore. We analyzed associations between THBS2 and ImmuneScore, and StromalScore in 33 types of cancers. We calculated the putative proportions of immune cells from gene expression profiles using the online analytical platform CIBERSORT (https://cibersort.stanford.edu/) [14]. We estimated the relationship between THBS2 and the relative abundance of tumor-infiltrating immune cells in 33 types of cancers using a reference set with 22 sorted immune cell subtypes (LM22) and CIBERSORT.

\section{Cell culture}

We cultured human stomach cancer (BGC-823) and human pancreatic cancer (PANC1) cells (China Center for Type Culture Collection, Wuhan, China) in RPMI 1640 medium, and cultured in Dulbecco Modified Eagle medium (DMEM). The cells were routinely maintained and supplemented with $10 \%$ fetal bovine serum.

\section{Transfection}

Cells were transfected with small interfering (si) THBS2 mRNA or negative control (NC) siRNA were designed and produced (Genepharma Shanghai, China) using the following forward and reverse $\left(5^{\prime}\right.$ " $\left.3^{\prime}\right)$ primers:

THBS2-SiRNA: GUUUGCUUCAGAACGUCCATT and UGGACGUUCUGAAGCAAACTT.

NC: UUCUUCGAACGUGUCACGUTT and ACGUGACAUGUUCGGAGAATT.

Thereafter, cancer cells were transfected with siTHBS2 or NC using Lipofectamine2000 (Thermo Fisher Scientific Inc., Waltham, MA, USA). 


\section{Cell migration, invasion, and proliferation assay}

We evaluated the migration and proliferative capacity of PANC1 and BGC-823 cells using CCK-8, transwell, and wound healing assays.

\section{Western blotting}

Total protein from PANC1 and BGC-823 cells extracted using RIPA buffer was resolved by gel electrophoresis then electroblotted onto PVDF membranes. Non-specific antigen binding was blocked with $5 \%$ skim milk, then the membranes were incubated at $4^{\circ} \mathrm{C}$ with gentle shaking overnight with the primary antibodies: 1:500-diluted THBS2 (A8561) and 1:1,000-diluted CD47 (A1838; both from ABclonal Technology, Woburn, MA, USA), 1:100-diluted MMP-2 (sc-133061; Santa Cruz Biotechnology Inc., Dallas TX, USA), or 1:5,000-diluted GAPDH (60004-1-Ig; Proteintech Group Inc., Rosemont, IL, USA). The membranes were incubated on the following day with 1:5,000-diluted secondary antibodies (SA00001-1, SA00001-2; Proteintech Group Inc., Rosemont, IL, USA).

\section{Quantitative RT-PCR}

Quantitative real-time polymerase chain reaction (qRT-PCR) proceeded using the StepOne ${ }^{\mathrm{TM}}$ PCR Detection System (Life Technologies Corp., Carlsbad, CA, USA) and the forward and reverse (5' " 3') primers: GAPDH: CATCATCCCTGCCTCTACTGG and GTGGGTGTCGCTGTTGAAGTC; THBS2: TCCTGCTGGCTCTGTGGGTGT; TGTGTTCTCACTGATGGCGT.

\section{Statistical analysis}

Differences between normal and cancer tissues were compared using Student t-tests. Associations between THBS2 expression and patient survival were investigated using univariate survival analysis and Kaplan-Meier curves. Values with $P<0.05$ were considered statistically significant. The statistical significance of the data in vitro was determined using GraphPad Prism 7 (GraphPad Software Inc., San Diego, CA, USA).

\section{Results}

\section{Expression of THBS2 mRNA in pan-cancer}

We evaluated THBS2 expression in 34 normal tissues from the GTEx database. The results showed that THBS2 was significantly higher in the cervix, uterus, endometrium, and adipose tissue, and significantly lower in the skeletal muscle, cerebellum, pancreas, and stomach (Figure 1B). We further analyzed the mRNA expression of THBS2 in the Oncomine database to explore THBS2 expression in pan-cancer. The 
findings revealed that cancer groups, such as bladder, colorectal, breast, esophageal, gastric, leukemia, liver, lung, lymphoma, myeloma, ovarian, pancreatic, and brain and central nervous system cancer, as well as head and neck cancer, showed higher THBS2 expression than normal groups (Figure 1C). We used TIMER2 to analyze RNA sequencing data from the TCGA database to evaluate THBS2 expression in pancancer. The differential expression of THBS2 in tumor and normal tissues is shown in Figure 1D. THBS2 expression was significantly lower in CESC, $\mathrm{KICH}$, and UCEC than in normal tissues. However, in BRCA, COAD, CHOL, ESCA, KIRC, KIRP, LUAD, LUSC, PRAD, PEAD, and STAD, THBS2 expression was significantly higher than that in normal tissues. Then, we analyzed THBS2 expression in pan-cancer and normal tissues using GEPIA2, which contains data of 31 tumor tissues from the TCGA database, as well as the data of their corresponding normal tissues in the GTEx database. The results indicated that THBS2 expression was significantly higher in 17 cancers, mainly in BRCA, PAAD, and SARC, and significantly lower in 14 cancers, especially in CESC, UCEC, and UCS (Figure 1A). These findings reveal that THBS2 is aberrantly expressed in various cancers.

\section{Prognostic value of THBS2 in pan-cancer}

We investigated whether THBS2 expression is associated with patient prognosis in pan-cancer (OS, DSS, DFI, and PFI). We found that THBS2 expression was significantly associated with OS in ACC, BLCA, KIRC, KIRP, LGG, MESO, PAAD, STAD, SKCM, and UVM. (Figure 2A). Abundant THBS2 expression was associated with a worse OS in all of these types of cancer except SKCM (Figure 2B-J). The expression of THBS2 influenced DSS in ACC, KIRC, KIRP, LGG, MESO, PAAD, and UVM among 32 types of cancer (Figure 3A). Kaplan-Meier curves associated increased THBS2 expression with a poor prognosis of these seven types of cancer. (Figure 3B-H). Forest plots showed that THBS2 expression influenced DFI in ACC, CESC, LIHC, and PAAD (Figure 4A). Kaplan-Meier curves of DFI associated high THBS2 expression with a worse prognosis in CESC and PAAD (Figure 4B-C). We the correlations between THBS2 expression and PFI, which revealed that THBS2 expression influenced PFI in BRCA, COAD, DLBC, KICH, KIRC, MESO, PAAD, PRAD, and UVM (Figure 5A). Except for DLBC, increased expression of THBS2 negatively impacted PFI in these cancer types. (Figure 5B-I). Our findings indicated an association between THBS2 and the stages of ACC, BLCA, COAD, ESCA, KIRC, PAAD, READ, SKCM, STAD, and THCA (Figure 6A-D). These findings show that THBS2 expression is substantially linked to patient prognosis in various cancers, particularly ACC, KICH, KIRP, STAD, and PAAD, and to OS, DSS, DFI, and PFI.

Types of cancer: ACC, BLCA, COAD, ESCA, KIRC, PAAD, READ, SKCM, STAD and THCA.

\section{Expression of THBS2 is correlated with immune infiltration and immune checkpoint markers}

Immune cells play important roles in tumorigenesis and cancer development [15] and are closely associated with patient survival and prognosis [16]. Therefore, we obtained scores for 15 types of 
infiltrative immune cells in 21 types of cancer using R. The results are shown as heat maps. (Figure 7).

In addition to analyzing immune-infiltrating cells, we also used ImmuneScore and StromalScore to assess immune and matrix components in pan-cancer. The results indicated that THBS2 expression significantly and positively correlated with the StromalScore in ACC, BLCA, BRCA, CESC, CHOL, COAD, GBM, ESCA, DLBC, HNSC, KIRC, KICH, LIHC, KIRP, OV, MESO, LUAD, LUSC, READ, PCPG, PRAD, SARC, SKCM, PAAD, UCEC, TICA, UCS, TGCT, UVM, and STAD (Figure 8A). The expression of THBS2 also significantly and positively correlated with ImmuneScore in BRCA, BLCA, LIHC, COAD, KIRC, KICH, PRAD, PCPG, PAAD, OV LUSC, LUAD, SARC, READ, UCEC, THCA, STAD, SKCM, and UVM (Figure 8B).

Given that we identified a relationship between THBS2 expression and immune infiltration, we further analyzed correlations between THBS2 expression and 48 common immune checkpoint genes in 33 cancers. The results indicated that THBS2 expression in BLCA, BRCA, COAD, ESCA, KICH, KIRC, LGG, LIHC, LUAD, LUSC, OV, PAAD, PRAD, PCPG, READ, SKCM, THCA, THYM, UCEC, and UVM, correlated with > 30 immune checkpoint markers (Figure 9). These results indicated that THBS2 plays significant roles in tumor immunity and the matrix as an ECM protein.

\section{Protein-protein interaction network of THBS2 in cancer}

We created a PPI network for THBS2 using STRING to identify probable processes through which THBS2 contributes to cancer carcinogenesis. Figure 10 shows that THBS2 is closely associated with ECM proteins such as MMP-2 and immune proteins, such as CD47.

\section{Thrombospondin-2 promoted proliferation and metastasis in PAAD and STAD}

To verify our results and better understand the biological effects of THBS2 on PAAD and STAD, we knocked down THBS2 expression by transfecting PANC-1 and BGC-823 cells with THBS2 small interfering (si) RNA. Western blotting results showed decreased expression of CD47, MMP-2 and THBS2 expression (Figure 11A-D). The results of CCK8 assays showed that THBS2 downregulation inhibited PANC1 and BGC-823 cell proliferation (Figure 11E). Finally, transwell and wound healing assays showed that THBS2 knockdown also inhibited the metastasis and invasion of PANC1 and BGC-823 cells. (Figure 12). These results indicated that THBS2 promotes the growth and metastasis of PAAD and STAD.

\section{Discussion}

Pan-cancer analysis has recently attracted increasing interest because it can easily and economically analyze tumor similarities and differences, and thus provide suggestions for cancer prevention and treatment targets [17]. Thrombospondin-2 protein belongs to the thrombospondin family, and is considered as a vital regulator of tumorigenesis. A clinical study of patients with cancer in China has 
found significant heterogeneity in the distribution of serum THBS2 in various types of cancer compared with healthy control individuals [18]. Thrombospondin-2 might be a diagnostic marker of pancreatic, gastric, non-small-cell lung, and colorectal cancers [2, 3, 19-21].

Our analysis of data from TCGA and the GTEx database revealed the abnormal expression of THBS2 in 17 types of cancer compared with healthy tissues. Therefore, we further investigated the importance of THBS2 expression to the prognosis of patients and found that increased THBS2 expression was associated with a worse prognosis (OS, DSS, DFI, and PFI), especially those with ACC, KICH, KIRP, PAAD, and STAD. These results indicated that THBS2 could be a prognostic marker for ACC, KICH, KIRP, PAAD, and STAD.

The TME has become a prominent and important area in tumor research. The TME consists of immune cells, stromal cells, blood vessels, and the ECM [22]. Immune cells comprise infiltrating cells such as B, T, and other types of immune cells. Immune cells play dual roles in tumors. On the one hand, they can detect and kill tumor cells [22, 23], whereas on the other hand, they can allow tumor cells to evade immune surveillance and metastasize via various mechanisms [24-26]. For example, CD8+ T cells can recognize and kill cancer cells that express aberrant tumor antigens. In response to the actions of various cytokines and environments, CD4+T cells can differentiate into various cell subtypes and participate in the adaptive immune response. Although the TME contains fewer B than T cells, they are nevertheless important to tumor development [27]. In addition to immune-infiltrating cells, other types of immune cells have specific functions in the TME. Elevated levels of macrophage infiltration are related to a poor prognosis among patients with breast, lung, and gastric cancers[11, 28, 29]. Neutrophils are recruited to the TME early in tumor development and promote tumor cell apoptosis by releasing cytokines and reactive oxygen species (ROS). However, neutrophils can later stimulate angiogenesis by producing matrix metalloproteinases (MMPs) that ultimately promote tumor progression, local invasion, and consequently enhanced tumor growth[30]. In conclusion, immune cells play crucial roles in tumorigenesis and progression. The present study found that THBS2 expression was significantly related to the abundance of various immune cells in various types of tumors. We also determined the THSB2 expression was associated with > 30 immune checkpoint genes in 20 types of cancer. ImmuneScore and StromalScore are associated with the amount of immune and matrix components in the tumor microenvironment[31]. Our results showed that THBS2 expression positively correlated with ImmuneScore and StromalScore in 30 and 19 types of cancer, respectively. The ECM plays an important role in the TME. In addition to being a physical scaffold for cells, it is also a vital element in driving tumor cell spread. Matrix metalloproteinases comprise a family of zinc- and calcium-dependent proteases that digest ECM proteins and are essential for reshaping the ECM [27]. Furthermore, MMPs not only degrade ECM, they play crucial roles in mediating tumor angiogenesis, metastasis, and invasion. Among the MMP family, MMP-2 promotes tumor growth, tissue invasion, angiogenesis, and metastasis [32-34], and its overexpression might be associated with tumor progression and a poor of ovarian epithelial carcinoma, oral cavity cancers, and non-small cell lung cancer [35-38]. Therefore, MMP-2 is considered a potential biomarker of tumorigenesis, a key effector of ECM remodeling, and a potential target for antitumor therapy. We showed that THBS2 in the tumor microenvironment was significantly associated with ECM 
proteins, including MMP-2, and immune-related proteins such as CD47, in the PPI network. Finally, our findings in vitro confirmed that THBS2 knockdown inhibited the proliferation, migration and invasion of PANC1 and BGC-823 cells and decreased the expression of the immune-related protein CD47 and the ECM proteinase MMP-2. These results suggest that THBS2 acts as a bridge between ECM and immune infiltration, thus influencing tumor progression.

\section{Conclusions}

In general, our findings indicated that THBS2 overexpression correlates with a poor prognosis and increased immune cell infiltration in numerous types of cancer. Furthermore, THBS2 was closely associated with various ECM and immune proteins, and the expression of immune checkpoint markers in various types of cancer. Our result in vitro confirmed that THBS2 knockdown inhibited CD47 and MMP-2 expression and the progression of pancreatic and gastric cancers. Thus, THBS2 might promote cancer progression, function as a bridge between the ECM and immune infiltration in cancer, and serve as a potential prognostic biomarker of several cancers, especially pancreatic and gastric adenocarcinoma.

\section{Abbreviations}

DFI: Disease-free interval

DSS: Disease-specific survival

ECM: Extracellular matrix

GEPIA2: Gene Expression Profiling Interactive Analysis

GTEx: Genotype-tissue expression

KM: Kaplan-Meier

MMPs: Matrix metalloproteinases

OS: Overall survival

PFI: Progression free interval

TAMs: Tumor-associated-macrophages

THBS2: Thrombospondin-2

TIMER2: Tumor immune estimation resource

TME: Tumor microenvironment 


\section{Declarations}

\section{Acknowledgements}

Not applicable.

\section{Authors' contributions}

XL: study design, experiments in vitro, data analysis, figure and table preparation, manuscript writing. WW: study design, data collection and analysis, manuscript writing, review and revision. BY: Manuscript reviewed and revision. ST: Manuscript reviewed and revision. All authors read and approved the final manuscript.

\section{Data availability}

The datasets used and analyzed during the current study are available from the corresponding author on reasonable request.

\section{Funding}

Not applicable.

\section{Ethics approval and consent to participate}

Not applicable.

\section{Consent for publication}

Not applicable.

\section{Competing interests}

The authors declare that they have no competing interests.

\section{Acknowledgements}

Not applicable. 


\section{Footnotes}

Xingchen Liao and Wei Wang are co-first authors. Both authors contribute equally to this work.

Baoping Yu and Shiyun Tan are corresponding authors.

\section{References}

1. Adams JC, Lawler J. The thrombospondins. Int J Biochem Cell Biol. 2004;36(6):961-8.

2. Berger AW, Schwerdel D, Reinacher-Schick A, Uhl W, Algul H, Friess H, et al. A Blood-Based Multi Marker Assay Supports the Differential Diagnosis of Early-Stage Pancreatic Cancer. Theranostics. 2019;9(5):1280-7.

3. Li L, Dong J, Fu L, Xia X, Pan F, Ning Y. Clinical Value of Serum Thrombospondin-2 Combined with CA19-9 in Early Diagnosis of Gastric Cancer. J Oncol. 2021;2021:2483964.

4. Wang X, Zhang L, Li H, Sun W, Zhang H, Lai M. THBS2 is a Potential Prognostic Biomarker in Colorectal Cancer. Sci Rep. 2016;6:33366.

5. Bindea G, Mlecnik B, Tosolini M, Kirilovsky A, Waldner M, Obenauf AC, et al. Spatiotemporal dynamics of intratumoral immune cells reveal the immune landscape in human cancer. Immunity. 2013;39(4):782-95.

6. Zhu Y, Yang J, Xu D, Gao XM, Zhang Z, Hsu JL, et al. Disruption of tumour-associated macrophage trafficking by the osteopontin-induced colony-stimulating factor-1 signalling sensitises hepatocellular carcinoma to anti-PD-L1 blockade. Gut. 2019;68(9):1653-66.

7. De Palma M, Biziato D, Petrova TV. Microenvironmental regulation of tumour angiogenesis. Nat Rev Cancer. 2017;17(8):457-74.

8. Cully M. Cancer: Re-educating tumour-associated macrophages with nanoparticles. Nat Rev Drug Discov. 2018;17(7):468.

9. He TF, Yost SE, Frankel PH, Dagis A, Cao Y, Wang R, et al. Multi-panel immunofluorescence analysis of tumor infiltrating lymphocytes in triple negative breast cancer: Evolution of tumor immune profiles and patient prognosis. PLoS One. 2020;15(3):e0229955.

10. Bocchialini G, Lagrasta C, Madeddu D, Mazzaschi G, Marturano D, Sogni F, et al. Spatial architecture of tumour-infiltrating lymphocytes as a prognostic parameter in resected non-small-cell lung cancer. Eur J Cardiothorac Surg. 2020;58(3):619-28.

11. Gordon SR, Maute RL, Dulken BW, Hutter G, George BM, McCracken MN, et al. PD-1 expression by tumour-associated macrophages inhibits phagocytosis and tumour immunity. Nature. 2017;545(7655):495-9.

12. Cheng Y, Wang T, Lv X, Li R, Yuan L, Shen J, et al. Detection of PD-L1 Expression and Its Clinical Significance in Circulating Tumor Cells from Patients with Non-Small-Cell Lung Cancer. Cancer Manag Res. 2020;12:2069-78. 
13. Chocarro de Erauso L, Zuazo M, Arasanz H, Bocanegra A, Hernandez C, Fernandez G, et al. Resistance to PD-L1/PD-1 Blockade Immunotherapy. A Tumor-Intrinsic or Tumor-Extrinsic Phenomenon? Front Pharmacol. 2020;11:441.

14. Newman AM, Steen CB, Liu CL, Gentles AJ, Chaudhuri AA, Scherer F, et al. Determining cell type abundance and expression from bulk tissues with digital cytometry. Nat Biotechnol. 2019;37(7):77382.

15. de Visser KE, Eichten A, Coussens LM. Paradoxical roles of the immune system during cancer development. Nat Rev Cancer. 2006;6(1):24-37.

16. Bonanno L, Pavan A, Dieci MV, Di Liso E, Schiavon M, Comacchio G, et al. The role of immune microenvironment in small-cell lung cancer: Distribution of PD-L1 expression and prognostic role of FOXP3-positive tumour infiltrating lymphocytes. Eur J Cancer. 2018;101:191-200.

17. Schaub FX, Dhankani V, Berger AC, Trivedi M, Richardson AB, Shaw R, et al. Pan-cancer Alterations of the MYC Oncogene and Its Proximal Network across the Cancer Genome Atlas. Cell Syst. 2018;6(3):282-300 e2.

18. Zou S, Li J, Yan J, Xu J, Lin M, Cao D. Distribution of serum Thrombospondin-2, a novel tumor marker, in general population and cancer patients in China. Clin Chim Acta. 2021;518:123-7.

19. Peng HY, Chang MC, Hu CM, Yang HI, Lee WH, Chang YT. Thrombospondin-2 is a Highly Specific Diagnostic Marker and is Associated with Prognosis in Pancreatic Cancer. Ann Surg Oncol. 2019;26(3):807-14.

20. Jiang YM, Yu DL, Hou GX, Jiang JL, Zhou Q, Xu XF. Serum thrombospondin-2 is a candidate diagnosis biomarker for early non-small-cell lung cancer. Biosci Rep. 2019;39(7).

21. Fei W, Chen L, Chen J, Shi Q, Zhang L, Liu S, et al. RBP4 and THBS2 are serum biomarkers for diagnosis of colorectal cancer. Oncotarget. 2017;8(54):92254-64.

22. Junttila MR, de Sauvage FJ. Influence of tumour micro-environment heterogeneity on therapeutic response. Nature. 2013;501(7467):346-54.

23. Morvan MG, Lanier LL. NK cells and cancer: you can teach innate cells new tricks. Nat Rev Cancer. 2016;16(1):7-19.

24. Dunn GP, Bruce AT, Ikeda H, Old LJ, Schreiber RD. Cancer immunoediting: from immunosurveillance to tumor escape. Nat Immunol. 2002;3(11):991-8.

25. Tang F, Xu Y, Zhao B. NLRC5: new cancer buster? Mol Biol Rep. 2020;47(3):2265-77.

26. Simiczyjew A, Dratkiewicz E, Mazurkiewicz J, Zietek M, Matkowski R, Nowak D. The Influence of Tumor Microenvironment on Immune Escape of Melanoma. Int J Mol Sci. 2020;21(21).

27. Anderson NM, Simon MC. The tumor microenvironment. Curr Biol. 2020;30(16):R921-R5.

28. Zhang H, Li R, Cao Y, Gu Y, Lin C, Liu X, et al. Poor Clinical Outcomes and Immunoevasive Contexture in Intratumoral IL-10-Producing Macrophages Enriched Gastric Cancer Patients. Ann Surg. 2020.

29. Choi J, Gyamfi J, Jang H, Koo JS. The role of tumor-associated macrophage in breast cancer biology. Histol Histopathol. 2018;33(2):133-45. 
30. Jaillon S, Ponzetta A, Di Mitri D, Santoni A, Bonecchi R, Mantovani A. Neutrophil diversity and plasticity in tumour progression and therapy. Nat Rev Cancer. 2020;20(9):485-503.

31. Bi KW, Wei XG, Qin XX, Li B. BTK Has Potential to Be a Prognostic Factor for Lung Adenocarcinoma and an Indicator for Tumor Microenvironment Remodeling: A Study Based on TCGA Data Mining. Front Oncol. 2020;10:424.

32. Chou KY, Chang AC, Ho CY, Tsai TF, Chen HE, Chen PC, et al. Thrombospondin-4 promotes bladder cancer cell migration and invasion via MMP2 production. J Cell Mol Med. 2021.

33. Ni X, Xia T, Zhao Y, Zhou W, Wu N, Liu X, et al. Downregulation of miR-106b induced breast cancer cell invasion and motility in association with overexpression of matrix metalloproteinase 2 . Cancer Sci. 2014;105(1):18-25.

34. Chou KY, Chang AC, Tsai TF, Lin YC, Chen HE, Ho CY, et al. MicroRNA34a5p serves as a tumor suppressor by regulating the cell motility of bladder cancer cells through matrix metalloproteinase 2 silencing. Oncol Rep. 2021;45(3):911-20.

35. Qian Q, Wang Q, Zhan P, Peng L, Wei SZ, Shi Y, et al. The role of matrix metalloproteinase 2 on the survival of patients with non-small cell lung cancer: a systematic review with meta-analysis. Cancer Invest. 2010;28(6):661-9.

36. Hoffmann C, Vacher S, Sirven P, Lecerf C, Massenet L, Moreira A, et al. MMP2 as an independent prognostic stratifier in oral cavity cancers. Oncoimmunology. 2020;9(1):1754094.

37. Shi Y, Su C, Hu H, Yan H, Li W, Chen G, et al. Serum MMP-2 as a potential predictive marker for papillary thyroid carcinoma. PLoS One. 2018;13(6):e0198896.

38. Jia H, Zhang Q, Liu F, Zhou D. Prognostic value of MMP-2 for patients with ovarian epithelial carcinoma: a systematic review and meta-analysis. Arch Gynecol Obstet. 2017;295(3):689-96.

\section{Figures}

\section{Figure 1}

Expression of THBS2 mRNA in pan-cancer.

A Differential expression of THBS2 in cancers and normal tissues determined using GEPIA2. B THBS2 expression in 34 normal tissues determined using GTEx database. C Comparison of THBS2 expression between normal and cancer tissues in ONCOMINE. D Expression of THBS2 in various cancer types according to TIMER2.

\section{Figure 2}


Correlations between THBS2 expression and overall survival.

A Relationship between HRs and THBS2 expression in 33 types of cancer. B-J Kaplan-Meier curves show relationships between differential expression of THBS2 and overall survival of ACC, BLCA, KIRC, KIRP, LGG, MESO, PAAD, STAD, SKCM and UVM.

\section{Figure 3}

Correlation between THBS2 expression and disease-specific survival.

A Relationships between hazard ratios and THBS2 expression in 32 cancers. B-H Kaplan-Meier curves show relationships between differential expression of THBS2 and disease-specific survival of ACC, KIRC, KIRP, LGG, MESO, PAAD and UVM.

\section{Figure 4}

Correlations between THBS2 expression and disease-free interval.

A Relationships between hazard ratios and THBS2 expression in 28 types of cancer. B-C Kaplan-Meier curves show relationships between differential expression of THBS2 and disease-free intervals in CESC and PAAD.

\section{Figure 5}

Correlations between THBS2 expression and platinum-free interval.

A Relationships between hazard ratios and THBS2 expression in 32 types of cancer. B-I Kaplan-Meier curves show relationships between differential expression of THBS2 and platinum-free intervals in BRCA, COAD, KICH, KIRC, MESO, PAAD, PRAD, and UVM.

\section{Figure 6}

Association between THBS2 expression and stages of 10 types of cancer.

Types of cancer: ACC, BLCA, COAD, ESCA, KIRC, PAAD, READ, SKCM, STAD and THCA. 


\section{Figure 7}

Correlations between THBS2 expression and abundance of infiltrating immune cells among types of cancer.

\section{Figure 8}

Correlations between THBS2 expression and ImmuneScore/StromalScore in cancers.

A Correlations between THBS2 expression and ImmuneScore were identified in ACC, BLCA, BRCA, CESC, CHOL, COAD, GBM, ESCA, DLBC, HNSC, KIRC, KICH, LIHC, KIRP, OV, MESO, LUAD, LUSC, READ, PCPG, PRAD, SARC, SKCM, PAAD, UCEC, TICA, UCS, TGCT, UVM, and STAD. B Correlations between THBS2 expression and StromalScore were identified in BLCA, BRCA, LIHC, COAD, KIRC, KICH, PRAD, PCPG, PAAD, OV, LUSC, LUAD, SARC, READ, UCEC, THCA, STAD, SKCM, and UVM.

\section{Figure 9}

Correlations between THBS2 expression and 48 common immune checkpoint genes in 33 types of cancer.

${ }^{\star} \mathrm{P}<0.05, \star \star \mathrm{P}<0.01, \star \star \star \mathrm{P}<0.001$

\section{Figure 10}

Protein-protein interaction network for THBS2.

\section{Figure 11}

Expression of THBS2 in PANC1 and BGC-823 cells.

A-C Western blots of PANC1 and BGC-823 cells with THBS2 knockdown. D Transfection efficiency of siRNA of THBS2 in PANC1 and BGC-823 cells validated by qRT-PCR. ${ }^{\star} P<0.05, * * P<0.01,{ }^{\star} * \star P<0.001$.

\section{Figure 12}


Thrombospondin-2 promotes proliferation and metastasis of PANC1 and BGC-823 cells.

A-F Migration and invasion of PANC1 and BGC-823 cells with THBS2 knockdown. G Proliferation of PANC1 and BGC-823 cells with THBS2 knockdown for 24 h. ${ }^{\star} \mathrm{P}<0.05,{ }^{*} \mathrm{P}<0.01,{ }^{*} * \mathrm{P}<0.001$. 